\title{
Special Issue on Recent Progress in Nonlinear Theory and Its Applications
}

The 2015 International Symposium on Nonlinear Theory and Its Applications (NOLTA2015), held in Hong Kong on 1-4 December 2015, was a great success. During the Symposium, many researchers and students exchanged their new ideas and discussed state-of-the-art research progress and results related to nonlinear science and engineering. As a subsequent program associated with the Symposium, this special issue of the NOLTA journal has been organized. We received 10 submissions from authors in a wide range of scientific fields. Thanks to the very active and professional Guest Associate Editors, the entire paper review process has been seriously and smoothly completed. As a result of rigorous evaluations, 7 papers were accepted. These excellent contributions are ranged from theoretical analyses to practical design and engineering applications, including such as converters, neural circuits, nonlinear filters, sensor networks, synchronizations, time-series and signal sequences.

We would like to express our sincere thanks to the Guest Associate Editors and reviewers for their time and efforts devoted to the reviews. We would also like to extend our appreciation to the Secretaries, Dr. Takuji Kousaka and Dr. Haruna Matsusita, for their hard work and efficient service.

\section{Guanrong Chen and Tetsushi Ueta Guest Editors}

\section{Editorial Committee of the Special Issue}

\section{Guest Editors:}

Guanrong Chen (City University of Hong Kong, China)

Tetsushi Ueta (Tokushima University, Japan)

\section{Guest Associate Editors:}

Tetsuya Asai (Hokkaido University, Japan)

Nelson Chan (City University of Hong Kong, China)

Ahmed Elwakil (University of Sharjah, UAE)

Kenya Jinno (Nippon Institute of Technology, Japan)

Francis C. M. Lau (Hong Kong Polytechnic University, China)

Zhong Li (Fern University-Hagen, Germany)

Wei Lin (Fudan University, China)

Jorge L. Moiola (Universidad Nacional del Sur, Argentina)

Maciej Ogorzalek (Jagiellonian University, Poland)

Michael Small (The University of Western Australia, Australia)

Isao Tokuda (Ritsumeikan University, Japan)

Wallace K.S. Tang (City University of Hong Kong, China)

Michael Tse (Hong Kong Polytechnic University, China)

\section{Secretaries:}

Takuji Kousaka (Oita University, Japan)

Haruna Matsushita (Kagawa University, Japan) 